
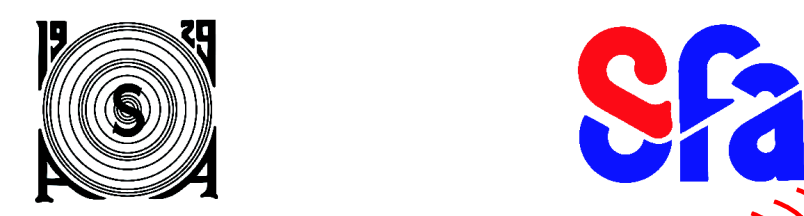

EAA

$\mathbf{r}$

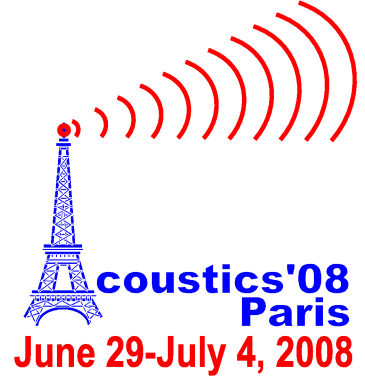

www.acoustics08-paris.org

\title{
euronoise
}

\section{A comparison between modeled and measured high frequency bottom backscattering}

\author{
D. G Simons ${ }^{\mathrm{a}}$ and M. Snellen ${ }^{\mathrm{b}}$
}

a Delft University of Technology, P.O. Box Postbus 5048, 2600 GA Delft, Netherlands ${ }^{b}$ Delft Institute of Earth Observation and Space Systems, Delft University of Technology, Kluyverweg 1, 2629 HS Delft, Netherlands m.snellen@tudelft.nl 
Compared to taking many sediment grabs, classification techniques based on acoustic remote sensing of the seafloor allow for significant cost reductions, providing the required information at much denser spatial scale. An attractive approach hereto is to use the multibeam-echosounder (MBES) which measures sediment backscatter strengths as a function of angle. By maximizing the match between this backscatter strength and model results, sediment properties can be derived. The backscatter model used here accounts for both surface roughness and volume scattering. MBES measurements $(300 \mathrm{kHz})$ were taken in the Cleaver-Bank area (North Sea), characterized by a wide variety of sediment types. For the majority of the sediment types occurring, welldefined single-sediment-type areas can be discerned. Based on the MBES measurements in these areas and corresponding geotechnical sediment parameters provided by grab grain size analysis, calibration curves are derived, representing the mismatch between modeled and measured backscatter strengths. These curves are found to be similar for all sediment types considered. By applying these correction curves to the MBES measurements, they show good agreement with the model for all observed sediment types. It is shown that after this correction the model can be used for sediment classification purposes.

\section{Introduction}

This paper deals with high-frequency backscatter strength measurements taken by a multibeam-echosounder (MBES) system which measures sediment backscatter strengths as a function of angle. The area in which the measurements were taken is the Cleaverbank area (North Sea), featuring a large number of different seafloor types. In this paper backscatter strengths as predicted by a model are compared with the Cleaverbank measurements of the backscatter strength. The availability of backscatter models, predicting backscatter strength as a function of seafloor type, allows for converting the measured backscatter strengths to seafloor properties. In general, however, backscatter measurements as provided by the MBES systems still contain system characteristics. A proper calibration of the measured backscatter strengths is therefore required, when comparing model output and measurements for classification purposes. Section 2 presents a description of the dataset, whereas in section 3 a short description of the backscatter model is given. Section 4 reports on the modeldata comparison. The paper ends with the summary and conclusions in section 5 .

\section{Description of the dataset}

The experiments were conducted in the North Sea. The selected area includes a variety of sediment types from soft and smooth ('sandy mud') to hard and rough ('sandy gravel'). The water depth ranges from $30 \mathrm{~m}$ in the gravel area to $60 \mathrm{~m}$ in the mud area. The experiments were carried out with ships equipped with a dual head $300 \mathrm{kHz}$ MBES system, capable of forming in total 254 beams, i.e., 127 beams for each of the two sonar heads.

For obtaining up-to-date information of the surface sediment of the sea bottom, a series of bottom grabs were taken along the acoustic tracks. Median values $d_{50}$ of the grain size distributions were determined, with $d_{50}$ being defined such that $50 \%$ of the grains, by weight, are smaller than $d_{50}$. In the following use will be made of $d_{50}$ to characterize the sediments, where it will be expressed as a value for $M_{\mathrm{z}}$, with $M_{z}=-{ }^{2} \log \left[d_{50}\right]$, where it is assumed that the mean and median grain size coincide. $M_{\mathrm{z}}$ values typically range from 9 to -1 . In the trial area under consideration we find values for $M_{\mathrm{z}}$ ranging from 5 to -1 .
In addition to the above, also sediment classification according to Folk [1] was assigned to each bottom grab. The Folk class is based on the relative amount of gravel, sand and mud (mud being silt or clay). The distinction between mud and sand is made on the basis of the grain size (diameter). All grains smaller than $62.5 \mu \mathrm{m}$ are assigned to the mud content of the bottom grab. Table 1 specifies for each of the Folk classes in the area, the corresponding $M_{z}$ values. Both Folk class and $M_{z}$ were determined from the core analysis. For the grabs that do contain gravel the values for the grain size are based on the Folk class and are taken from [2].

\begin{tabular}{|c|c|}
\hline Folk class & $M_{z}$ \\
\hline $\mathrm{sG}$ & -1 \\
\hline $\mathrm{gS}$ & 0.5 \\
\hline $\mathrm{S}$ & $1-2$ \\
\hline $\mathrm{mS}$ & $2-3.5$ \\
\hline $\mathrm{sM}$ & $4.5-5$ \\
\hline
\end{tabular}

Table $1 M_{z}$ for each of the Folk classes.

Ref. [3] presents a method for seafloor classification using MBES data. The method employs the MBES backscatter data. Applying the Bayes multiple decision rule, the method discriminates between sediments in the most optimal way, such that it accounts for the ping-to-ping variability of the backscatter intensity that partly masks the information about seafloor characteristics. The backscatter data employed consist of data at a single beam angle. The backscatter data have been obtained by correcting for propagation losses and footprint size. However, the angular dependence is not eliminated. In [3] also the results of applying the method to the current dataset is presented. Comparison of these classification results with the core data and with the geological map indicates that the method is capable of discriminating between areas with different seafloor types. Seafloor types are assigned to the different areas, based on a comparison with the cores and the geological map. Fig. 1 shows the resulting map, together with the core data. The classification indicated the presence of six different seafloor types, i.e., sandy gravel (sG), gravelly sand (gS), slightly gravely sand ((g)S), sand (S), muddy sand $(\mathrm{mS})$, and sandy mud (sM). 


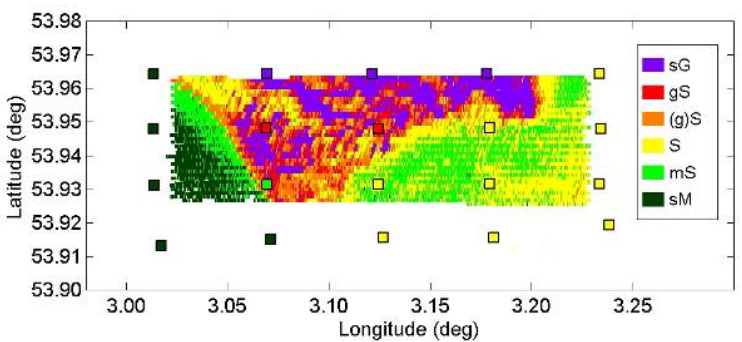

Fig. 1 Classification map based on MBES backscatter data taken at for the $60^{\circ}$ angle $\left(30^{\circ}\right.$ grazing angle). Also indicated are the grab Folk classes for comparison.

\section{The backscatter model used}

We have employed the backscatter model as described in [2]. The total backscatter strength is modelled as the result of a contribution from volume backscattering and rough interface backscattering, $B S(\theta)=10^{10} \log \left[\sigma_{r}(\theta)+\sigma_{v}(\theta)\right]$. Here, $\sigma_{r}$ and $\sigma_{v}$ are the backscattering cross sections, per unit area and per unit solid angle, due to the interface roughness and due to volume scattering, respectively.

$\sigma_{r}$ is modelled as the result of three different approximations ([3]): 1) The Kirchhoff approximation, valid for grazing incidence angles near $90^{\circ}$; 2) The composite roughness approximation, valid for smooth to moderately rough sediment and grazing incidence angles away from $90^{\circ}$; 3) Large-roughness scattering, where the scattering cross section is determined from an empirical expression which is derived for rough sediments, like gravel and rock. The resulting $\sigma_{r}$ is obtained by appropriate interpolation between these three approximations.

All three contributions are a function of the roughness spectrum. Following [2] the following isotropic relief spectrum is assumed: $W_{2}(K)=\left(h_{0} K\right)^{-\gamma} w_{2}$, with $K$ the wave number of the bottom relief, $h_{0}$ a reference length (1 $\mathrm{cm}), w_{2}$ the spectral strength parameter and $\gamma$ the spectral exponent. Additionally, the backscatter strengths are determined by the sediment density, attenuation coefficient and sound speed.

$\sigma_{v}$ is modelled (cf. [2]) based on the following expression for sediment volume backscattering cross section

$$
\sigma_{p v}=\frac{5 \delta \sigma_{2}\left|1-R^{2}(\theta)\right| \sin ^{2}(\theta)}{v \ln 10 \mid P(\theta)^{2} \operatorname{Im}\{P(\theta)\}}
$$

with $v$ the ratio of sediment sound speed to water sound speed, $\delta$ the ratio of imaginary wavenumber to real wavenumber for the sediment, $R$ the amplitude reflection coefficient, $\theta$ the grazing angle of incidence and $P(\theta)=\sqrt{\kappa^{2}-\cos ^{2} \theta}$, with $\kappa=\frac{1}{v}(1+i \delta) . \quad \sigma_{2}$ is the ratio of sediment volume scattering cross section to attenuation coefficient. $\sigma_{v}$ is determined from $\sigma_{p v}$ accounting for shadowing and bottom slope correction.

The parameters needed as input to the backscatter model are:
- $\quad \rho$ and $v$ : sediment - water ratios of mass density and sound speed;

- $\delta$ : ratio of imaginary wavenumber to real wavenumber for the sediment;

- $\quad \gamma$ and $w_{2}$ : exponent and strength of bottom relief spectrum;

- $\sigma_{2}$ : ratio of sediment volume scattering cross section to attenuation coefficient.

Empirical relations are available that relate $w_{2}, \rho, v$ and $\delta$ to sediment mean grain size [3]. $\gamma$ is taken as 3.25 .

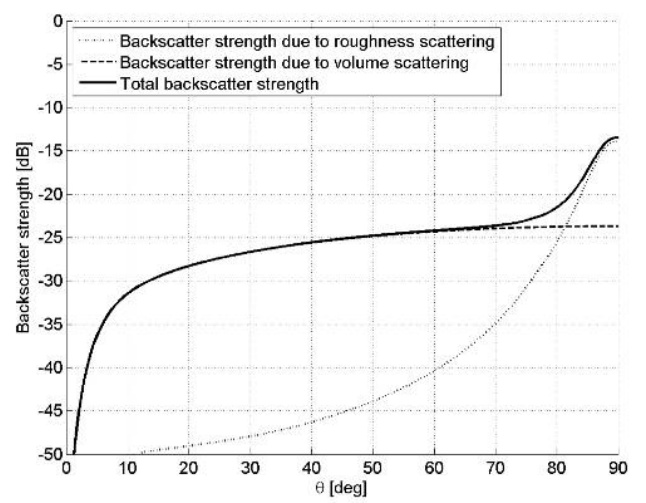

Fig. 2 Backscatter strength as predicted by the model for a sediment with $M_{z}=5$.

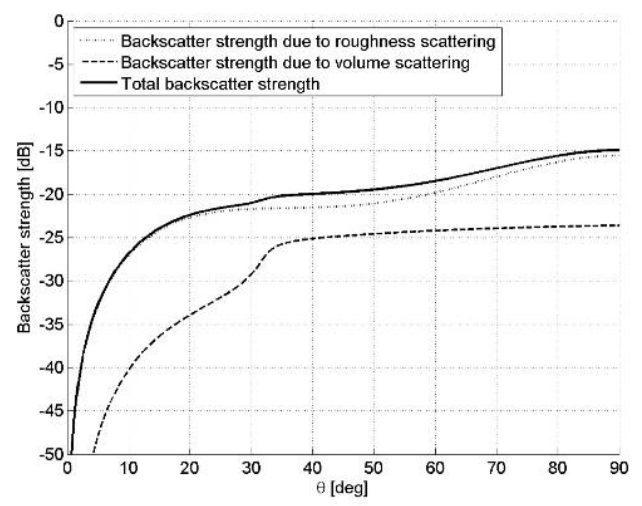

Fig. 3 Backscatter strength as predicted by the model for a sediment with $M_{z}=1.5$.

Figures 2-3 show examples of backscatter curves calculated by the model for values of $M_{z}$ of 5 and 1.5. From these plots it van be seen that for small grain sizes (high $M_{z}$ ), roughness scattering mainly contributes for angles close to nadir, whereas volume scattering dominates for the grazing angles. For the larger grain size sediments (small $M_{z}$ ), the main contribution to the total backscatter strength stems from roughness scattering due to the limited penetration into the sediment.

\section{$4 \quad$ Results}

For comparing the measured backscatter curves with those predicted by the model, areas with constant sediment type need to be selected. In addition, the water depth variations in these areas should be limited to avoid the effects of slope variations on the measured backscatter curves. 
This selection was carried out based on the acoustic classification results as shown in Fig. 1. For each of the different seafloor types, mean backscatter curves have been determined.

Figure 4 shows the resulting mean curves as solid lines, with colours indicating different seafloor types and taken equal to the colours used in Fig. 1. Also shown in this figure are the results of model calculations as dashed lines.

A)
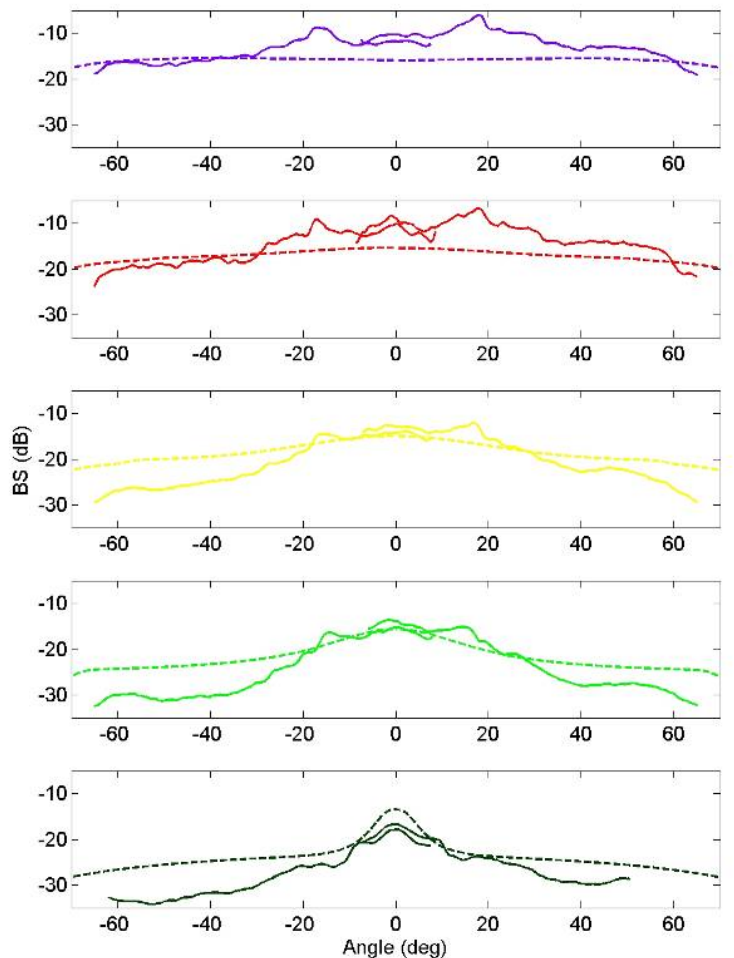

Mean BS: sG

B) -... Model results: $M_{z}=-1$ Mean BS: gS -.... Model results: $M_{z}=0.5$ Mean BS: $S$ Model results: $M_{z}=1.5$ -Mean BS: mS Model results: $M_{z}=3$ -Mean BS: $\mathrm{SM}$ -.... Model results: $M_{z}=5$

Fig. 4 A) Predicted backscatter strength curves (dashed lines) and measured backscatter strength curves (solid lines).

Fig. 4 B) Legend for Fig 5 A) and Fig. 6.

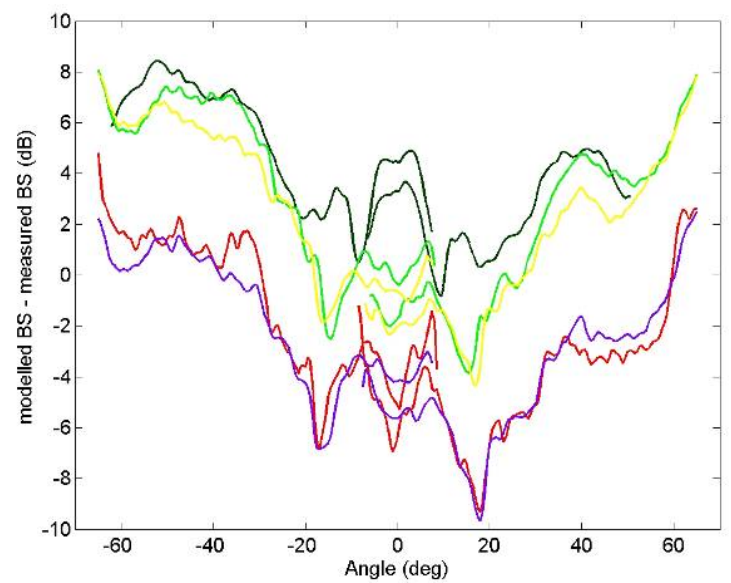

Fig. 5 Difference between modeled and measured backscatter strength.

$M_{z}$ values used for the model calculations are based on the values specified by Table $1: M_{z}=5$, for sandy mud, 3 for muddy sand, 1.5 for sand, 0.5 for gravelly sand and -1 for sandy gravel. Since no cores were taken for the slightly gravelly sand type of sediment, we have not considered this seafloor type in the analysis presented in this section. From this figure it can be seen that for some angular regions and sediment types, model and measurements show a good agreement, but that there are also significant differences.

Figure 5 shows for each of the sediment types considered the difference between model and measurements. It is seen that the differences are dependent on seafloor type. Consequently, these curves do not represent the calibration curve, representing the effects of system characteristics. Refinement of the model input, to make it representative for the area under consideration is needed.

In [2] it is stated that the model input parameters $w_{2}$ and $\sigma_{2}$ show a large scatter and therefore, these parameters can in practice deviate significantly from the values obtained by the parameterizations. For the model calculations shown in Fig. $4 \sigma_{2}$ is taken as 0.002 for all sediment types and $w_{2}$ is determined from the empirical expression relating it to the mean grain size. Considering Figs. 4 and 5, it is seen that for the small grain size sediments, i.e., sandy mud, muddy sand and sand, the model overestimates the backscatter strength for angles away from nadir. For the larger grain size sediments this is not the case. A first step, therefore, in improving the match between model and measurements is to decrease the volume scattering parameter $\sigma_{2}$. This lowers the predicted backscatter strength for the small grain size sediments for angles away from nadir, but hardly affects the predicted backscatter strength for the large grain size sediments. As a first test, parameter $\sigma_{2}$ has been decreased by a factor of 2. Figure 6 shows the resulting curves, whereas Fig. 7 presents the differences between model predictions and measurements. Clearly the model-data agreement has increased for the small grain size sediments. It can be seen that also the curves corresponding to the different seafloor types are closer together.
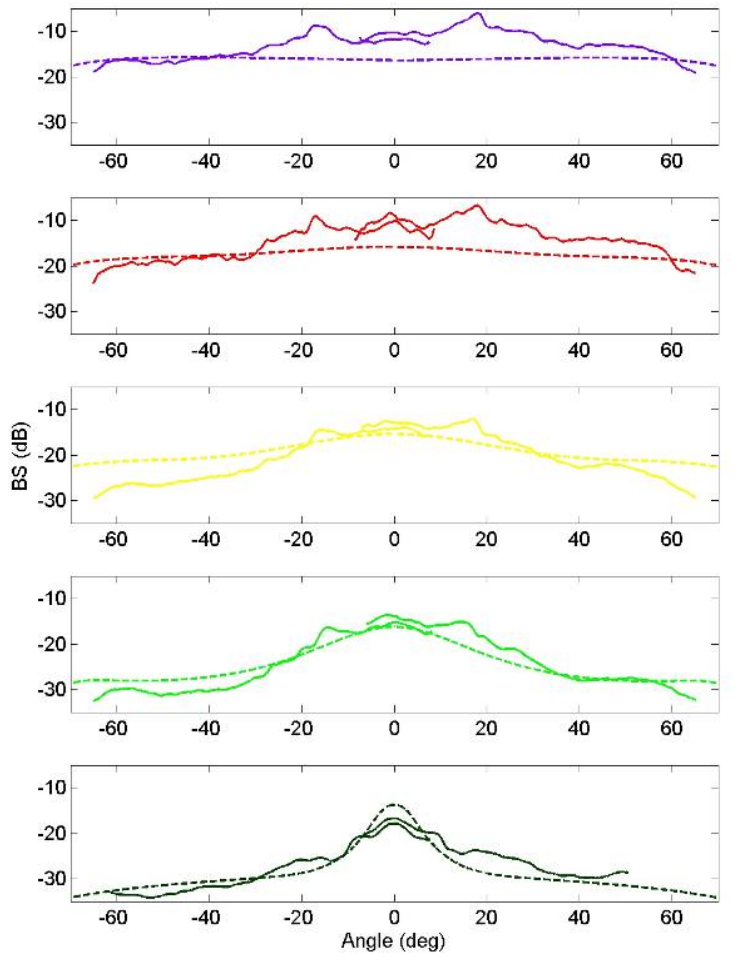

Fig. 6 Predicted backscatter strength curves (dashed lines) and measured backscatter strength curves (solid lines). 


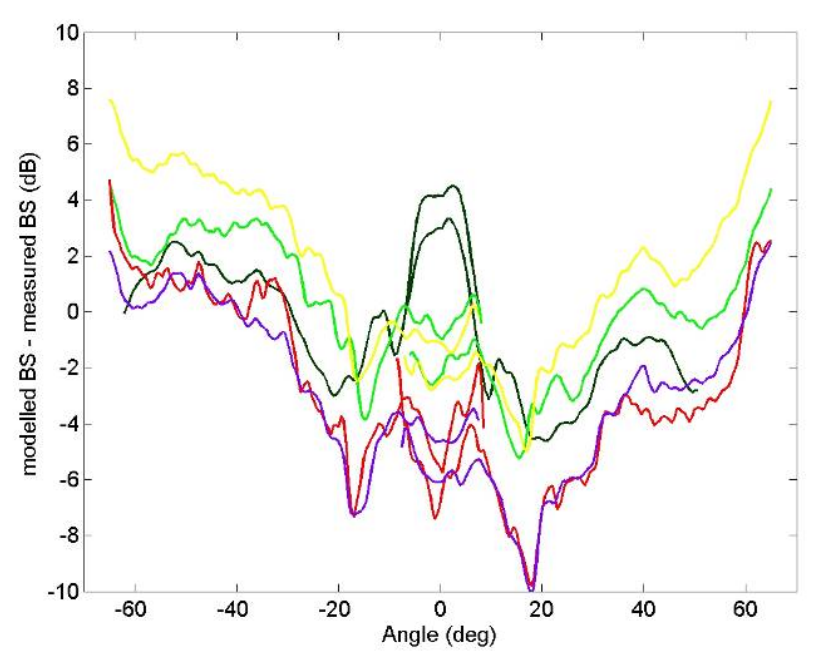

Fig. 7 Difference between modeled and measured backscatter strength.

In addition to the volume scattering parameter $\sigma_{2}$, also the roughness parameter $w_{2}$ is known to show deviations from the values predicted by the empirical relations. Therefore, a search was carried out for obtaining those values for $\sigma_{2}$ and $w_{2}$ that provide a maximum agreement between the modeldata differences for the seafloor types considered. The energy function was taken such that its minimum is obtained if the curves representing the model-data mismatch coincide for all seafloor types considered:

$$
E(\mathbf{x})=\sqrt{\left(\sum_{n}\left|S B_{\text {mod }, n}(\mathbf{x})-S B_{\text {meas }, n}\right|-|M|\right)^{2}}
$$

with $n$ indexing the different seafloor types, $S B_{\text {mod, } n}$ the modelled backscatter strengths and $S B_{\text {meas }, n}$ the measured backscatter strength. $M$ is the mean curve, i.e., the mean of the difference curves for all seafloor types considered.

The vector $\mathbf{x}$ contains the unknowns $\sigma_{2}$ and $w_{2}$ where $w_{2}$ is modelled as the $w_{2}$ obtained from the empirical expressions multiplied by an unknown multiplication factor. It should be noted that it is assumed that both unknown parameters are equal for all sediment types. For the optimization use was made of differential evolution. More details on this method can be found in [4]. The search bounds for the unknowns are listed in Table 2.

The resulting model-data differences are shown in Fig. 8. It can be concluded that the agreement between the different curves in Fig. 8 has improved compared to that shown in Fig. 7. Table 2 lists the resulting values for $\sigma_{2}$ and $w_{2}$, where $w_{2}$ is given as the value obtained from the empirical expressions, relating $w_{2}$ to mean grain size, multiplied by a factor. This factor is found to be 0.44 , i.e., to obtain a low function for the energy function the roughness should be taken less than half of its original value. The result is a decrease in backscatter strength for angles away from nadir, and an increase in backscatter strength for angles close to nadir. The decrease in the sediment volume scattering parameter results in an decrease in backscatter strength for angles away from nadir. Still the difference curves for the different seafloor types do not coincide.

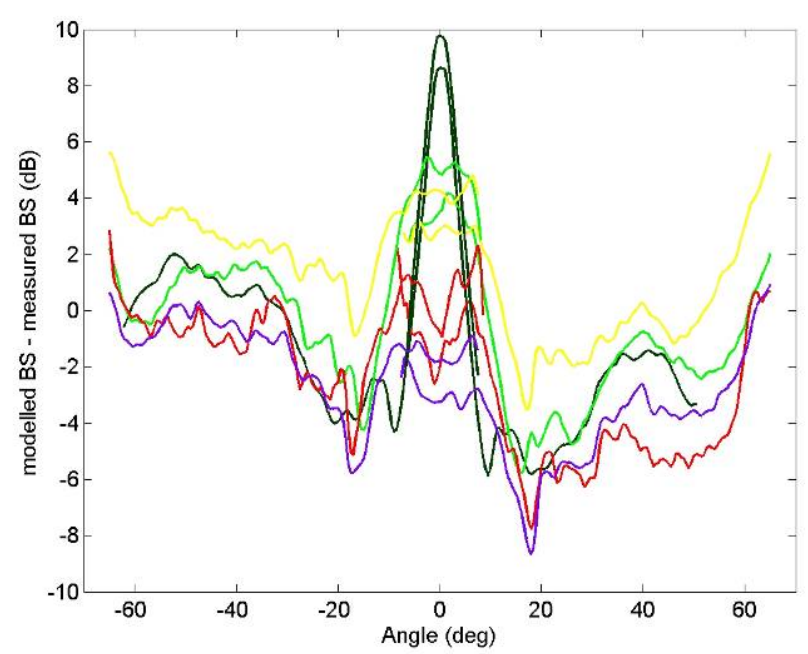

Fig. 8 Difference between modeled and measured backscatter strength after optimization for $\sigma_{2}$ and $w_{2}$.

From Table 1 it is seen that the mean grain size values for each of the sediment types show a spread. Therefore, as the final step in determining the calibration curve is to include also the values for $M_{z}$ in the optimization. The parameter values obtained are listed in Table 2 and the difference curves are shown in Fig. 9. Although the curves still do not coincide, they show very similar behaviour and the values are close. The calibration curve is taken as the mean of the individual curves and is shown as the thick solid black line in Fig. 9. The main deviations from this curve occur in the region close to nadir.

Figure 10 shows a comparison between the resulting model predictions and measured backscatter strengths. The dashed lines show the model predictions, with the different colours corresponding to different seafloor types. The thin solid lines show the measured mean backscatter curves. Shown as thick solid lines are the measured mean backscatter curves, but with corrections according to the calibration curve applied. It is seen that a satisfactory agreement with the model predictions is then obtained.

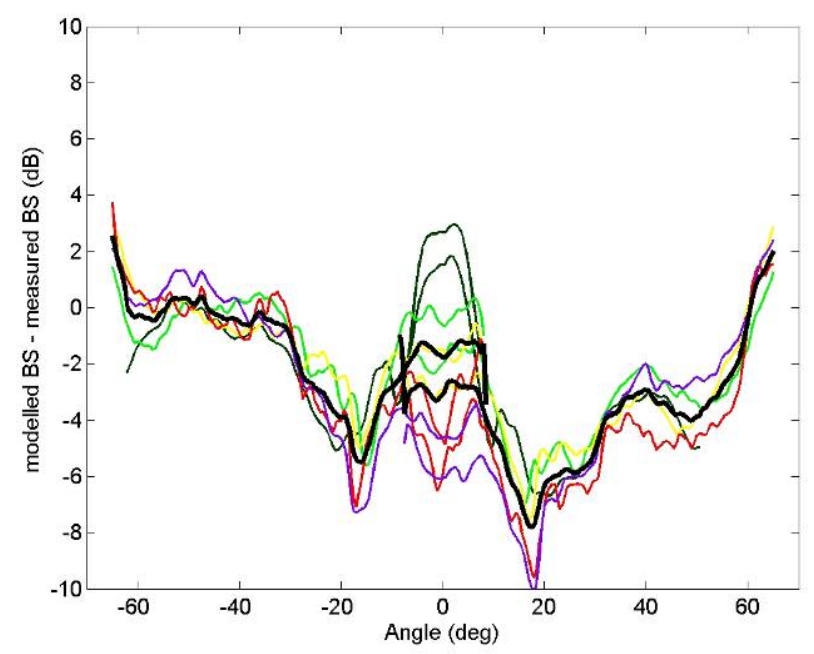

Fig. 9 Difference between modeled and measured backscatter strength after optimization for $M_{z}$ values and $\sigma_{2}$ and $w_{2}$. The thick black line is the mean curve and is taken as the calibration curve, representing the system characteristics. 

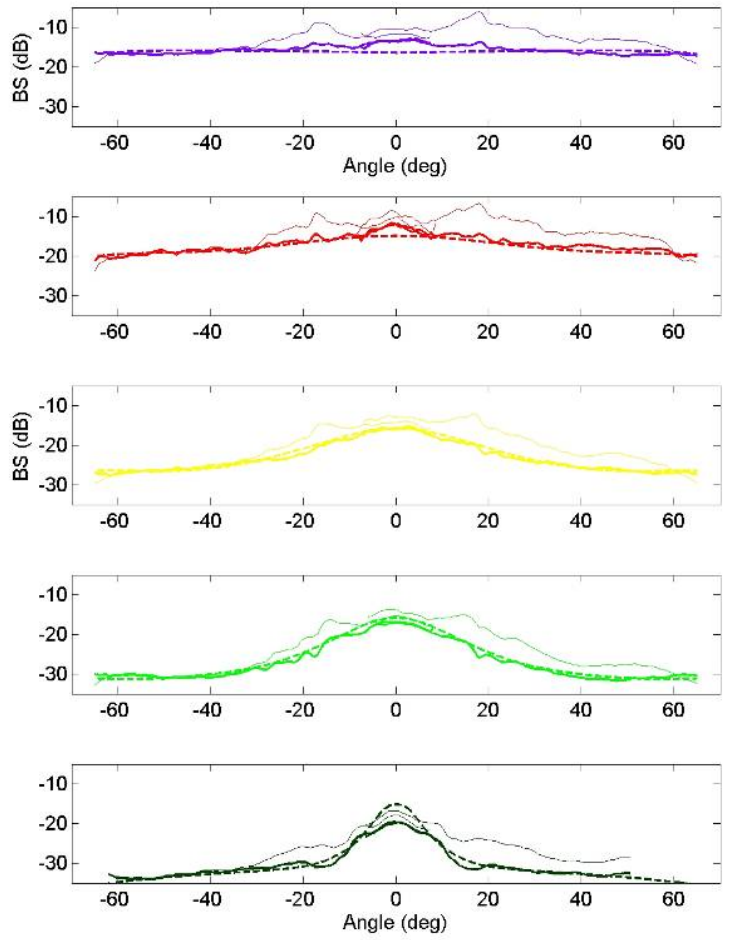

Fig. 10 Predicted backscatter strength curves (dashed lines) and measured backscatter strength curves (thin solid lines).

Thick solid lines represent the measured backscatter strength curves but with the calibration curve applied.

\begin{tabular}{|c|c|c|c|c|}
\hline $\begin{array}{c}\text { Para- } \\
\text { meter }\end{array}$ & \multicolumn{2}{|c|}{ Search bounds } & $\begin{array}{c}\text { Result of } \\
\text { optimization } \\
1\end{array}$ & $\begin{array}{c}\text { Result of } \\
\text { optimization } \\
2\end{array}$ \\
\hline$\sigma_{2}$ & 0.0001 & 0.004 & 0.0005 & 0.0003 \\
\hline$w_{2}$ & 0.2 & 2 & $0.44 w_{2, \mathrm{org}}$ & $0.96 w_{2, \mathrm{org}}$ \\
\hline$M_{z}$-sG & -1 & -0.5 & -1 & -1 \\
\hline$M_{z}$-gS & 0 & 1 & 0.5 & 1 \\
\hline$M_{z}$-S & 0.5 & 2.5 & 1.5 & 2.5 \\
\hline$M_{z}$-mS & 1.5 & 4 & 3 & 3.5 \\
\hline$M_{z}$-sM & 4 & 5.5 & 5 & 5.5 \\
\hline
\end{tabular}

Table 1 The unknown parameters, their search bounds and the results of the optimization. The numbers in italics in the column specifying the results of optimization 1 were used directly as input (not optimized).

As a final step model calculations for a range of $M_{z}$ values are carried out employing the optimized values for $\sigma_{2}$ and $w_{2}$. For each ping the backscatter strength curve, with the calibration applied, is compared to all predicted curves. The $M_{z}$ value resulting in the maximum match between measurement and model is assigned to the measurement position. Figure 11 shows the resulting grain size maps. Data taken by each of the two transducers is treated separately as a consistency check. Clearly the two maps show almost equal results and indicate areas differencing in seafloor type, comparable to those in Fig. 1.
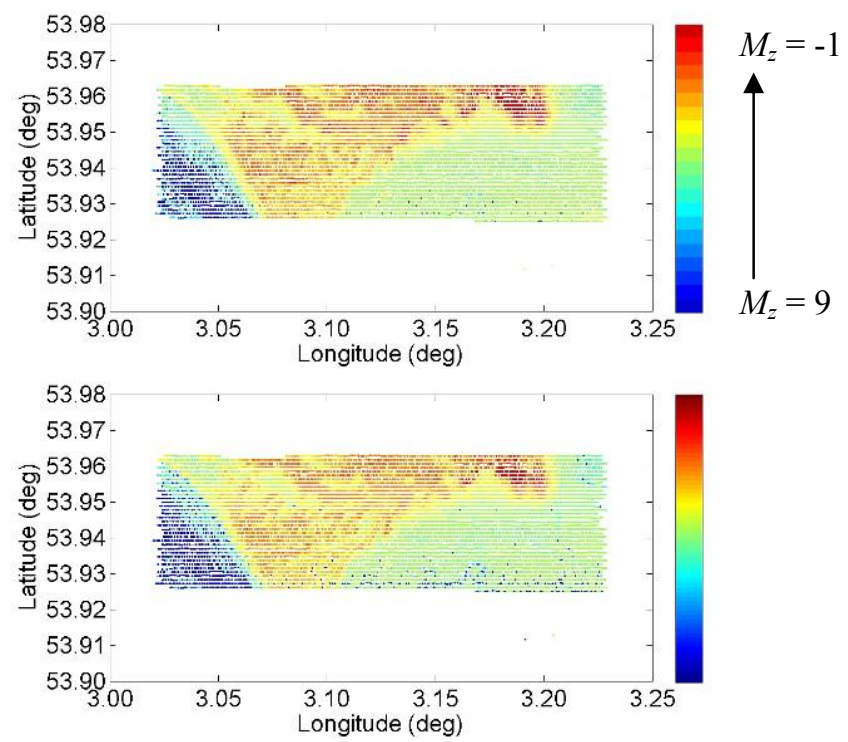

Fig. 11 Mean grain size maps for the two transducers. Colors indicate grain sizes from 9 to -1 in steps of $0.5 \varphi$.

\section{Conclusion}

Comparisons between predicted and measured backscatter strength curves are used to reveal the effects of system characteristics on the measured backscatter strengths. The data considered were taken with a $300 \mathrm{kHz}$ multibeamechosounder. The model employed predicts the backscatter strength as the result of roughness and volume backscatter. Employing the obtained calibration curve, representing the system characteristics, for seafloor classification, allows for directly estimating the sediment grain size for each transmitted ping.

\section{Acknowledgments}

This research is supported by the Dutch Technology Foundation STW, applied science division of NWO and the Technology Program of the Ministry of Economic Affairs.

\section{References}

[1] Robert L. Folk, "The distinction between grain size and mineral composition in sedimentary-rock nomenclature," Journal Geology 62, 344-359 (1954)

[2] APL-UW High-frequency Ocean Environmental Acoustic Models Handbook, Technical Report, APLUW TR 9407, AEAS 9501, October 1994

[3] Dick G. Simons and Mirjam Snellen, "A Bayesian approach to seafloor classification using multi-beam echo-sounder backscatter data", submitted to the Journal of Applied Acoustics

[4] Mirjam Snellen and Dick G. Simons, "An assessment of the performance of global optimisation methods for geo-acoustic inversion", accepted for publication in the Journal of Computational Acoustics 